\title{
Optimum Combinations of GGM and GDEM Models for Precise National Geoid Modelling
}

\author{
Essam Mohamed Al-Karargy*, Gomaa Mohamed Dawod \\ Department of physical geodesy, Survey Research Institute, National Water Research Center, Cairo, Egypt \\ Received 21 September 2020; received in revised form 17 December 2020; accepted 27 January 2021 \\ DOI: https://doi.org/10.46604/peti.2021.6452
}

\begin{abstract}
This study aims to develop a Local Geoid Model (LGM) for Egypt to determine the optimal combinations of global models with Global Navigation Satellite Systems (GNSS/Levelling) data. A precise national geodetic dataset, four Global Geopotential Models (GGMs), and three global Digital Elevation Models (DEMs) have been utilized. Hence, twelve gravimetric LGMs have been developed using the Least-Square Collocation (LSC) method fitted to GNSS/Levelling data and judged over 100 checkpoints. Results revealed that improvements in local geoid accuracy are attributed mainly to GGMs models representing the long wavelength of the Earth's gravitational field. Regarding DEMs, the accuracy of LGMs does not significantly depend on the utilized DEM. Based on the available data, the attained optimum geoid of Egypt has been developed with a standard deviation, equals $0.129 \mathrm{~m}$.
\end{abstract}

Keywords: GGM, DEM, GNSS, geoid, Egypt

\section{Introduction}

GNSS play a vital role in surveying geodetic activities worldwide. It produces geodetic or ellipsoidal heights reference to the surface of an ellipsoid, while most surveying and mapping activities depend on the orthometric heights referenced to the Mean Sea Level (MSL) datum. Hence, height conversion of GNSS heights to MSL heights necessitates a geoid model to define the vertical separation, geoid heights, between those two surfaces over a specific region. Geoid modelling, on a national scale, has been a crucial task for geodetic communities in several countries worldwide in the last few decades. For example, the U.S National Geodetic Survey (NGS) is currently developing a time-dependant geoid model for the entire USA to be completed by 2022 [1]. Similarly, an updated geoid model for the United Kingdom (UK) and Ireland has been developed to increase accuracy and homogeneity of height transformation across this region [2]. Additionally, The French Institut national de l'information géographique (IGN) is developing a new precise geoid model for France that could achieve a millimeter level of accuracy in height transformation [3]. More recently, the Geospatial Information Authority (GIA) of Japan is developing a new refined gravimetric geoid model on a 1x1.5 arc-second grid [4].

GGMs constitute a major factor in geoid modelling since they provide the long and medium wavelengths of the Earth's gravitational field. Since the mid-1960s, numerous GGMs have been developed and being applied in geoid modelling. Consequently, the evaluation of GGMs in a specific region has been extensively investigated by geodetic studies in the last few decades. For example, Fernádez et al. [5] and El-Ashquer et al. [6] have utilized free-air gravity anomalies and GNSS/Levelling datasets to study the selection of optimal GGM in Costa Rica and Kuwait respectively. Additionally, Gomez et al. [7] have utilized mean water level data in a lake in Argentina as a validation tool for GGM assessment. Since the Earth Geopotential Model 2008 (EGM2008) is the most well-known GGM worldwide, its precision performance has been

* Corresponding author. E-mail address: essamalkrargy@ gmail.com

Tel.: +002-10-66361763; Fax: +002-02-33933536 
investigated in several countries such as Egypt [8] and Sweden [9]. On the other hand, Global DEMs (GDEMs) have been utilized in geoid modelling for the evaluation of the terrain effects. So, the optimum DEM is also significant in such a task particularly in countries with no published precise DEM.

In Egypt, several geoid development tryouts have been carried out in the last few decades. Alnaggar [10] has developed the first pioneer national-scale geoid. Dawod [11] has developed a national geoid model based on the data of the Egyptian National Standardization Network of 1997 (ENGSN97) along with GPS/levelling data. Also, Abd-Elmotaal [12] has developed a gravimetric geoid model utilizing high-degree tailored reference geopotential model. Recently, Dawod and Abdel-Aziz [13] have studied the utilization of a Geographic Information Systems (GIS) technique in geoid modelling. The traditional manner of investigating the accuracy and reliability of GGMs and DEMs is the assumed point-wise comparison. In this approach, a specific GGM is evaluated over checkpoints with known geoidal undulations and the statistical indicators provide a measure for its reliability in geoid modelling [6]. Similarly, a particular DEM is judged over known stations with available orthometric heights.

A novel approach is proposed in the current study for deciding which GGM and DEM models are optimum for utilization in developing a national geoid model. Accordingly, this study aims at investigating the optimum combination of some selected GGMs and GDEMs models in developing a precise local geoid model of Egypt based on the available local heterogeneous geodetic datasets.

\section{Methodology}

The current study employs four GGMs models and three GDEMs models in developing twelve 5'x5' local geoid models for Egypt. GDEMs models have been utilized since there is no local precise DEM published for Egypt [14]. The utilized GGMs models include XGM2019e_2159, EGM2008, EIGEN-6C4, and GECO. These models have been selected since they are the only (to date) high-resolution GGMs with a degree of 2190. Concise descriptions of those models are presented in the following subsections:

\subsection{Global geopotential models (GGMs)}

(1) XGM2019e_2159: A recent GGM combines 15' terrestrial gravity anomalies datasets over land, 1' mean sea surface database over oceans, 5' GOCO06s GGM, and the EARTH2014 topographic model. It has been developed to degree 2190 (utilized herein) and later extended to degree 5400 [15].

(2) GECO: A global gravity model utilizes the GOCE satellite-based gravity data to improve the accuracy of the EGM2008 GGM in low and medium frequencies. GECO is developed, in 2015, up to degree and order 2190 [16]. GECO is the most-recent published high -resolution GGM up till 2017.

(3) EIGEN-6C4: A model released in 2014, that utilizes satellite tracking data (from the Laser Geodynamics Satellite: LAGEOS, the Gravity Recovery and Climate Experiment: GRACE, and the: Gravity-field and steady-state Ocean Circulation Explorer: GOCE missions) along with a global surface gravity anomaly grid and altimetry data. The model is up to 2190 degree, developed by both the Germany German Research Center for Geosciences (GFZ) and the French Centre National d'Études Spatiales (CNES) [17].

(4) EGM2008: An integrated GGM developed in 2008 by the U.S. National Geospatial-Intelligence Agency (NGA) up to 2190 degree, based on GRACE-based satellite tracking data, terrestrial gravity data, and altimetry data. It was a milestone in GGM development since its preceding model did not exceed 360 maximum degrees [18]. Table 1 shows the characteristics of those selected global GGMs. 
Table 1 Characteristics of selected GGMs

\begin{tabular}{|c|c|c|c|c|}
\hline GGM & Year & Degree & Utilized data & $\begin{array}{c}\text { Average accuracy over 12035 } \\
\text { GNSS/levelling stations (m) }\end{array}$ \\
\hline XGM2019e_2159 & 2019 & 2190 & A, G, S(GOCO06s), T & 0.236 \\
\hline GECO & 2015 & 2190 & EGM2008, S(GOCE) & 0.237 \\
\hline EIGEN-6C4 & 2014 & 2190 & A, G, S (GOCE, GRACE, Lageos) & 0.236 \\
\hline EGM2008 & 2008 & 2190 & A, G, S(GRACE) & 0.240 \\
\hline
\end{tabular}

\subsection{Global digital elevation models (GDEM)}

On the other hand, the utilized GDEMs models, herein, include SRTM1, ASTER, and ACE2 models. Table 2 summarizes the characteristics of those selected global DEMs, including:

(1) SRTM: The Shuttle Radar Topography Mission (SRTM) is a global DEM, that has two versions: SRTM1 with a spatial resolution of 1 arc second, i.e., approximately 30 meters, and SRTM3 model with a 3 arc second resolution. SRTMGL1 v. 3 [20] has been utilized herein. (download from e.g. https://earthexplorer.usgs.gov/).

(2) ACE2: The Altimeter Corrected Elevations, Version 2 is a global digital elevation model created by synergistically merging the SRTM data set with Satellite Radar Altimetry within the region bounded by $60^{\circ} \mathrm{N}$ and $60^{\circ} \mathrm{S}$. ACE2 was developed at resolutions of 3, 9 and 30 arc-seconds, and 5 arc-minutes [21]. The 3" ACE2, utilized herein (downloaded from https://sedac.ciesin.columbia.edu/data/set/dedc-ace-v2).

(3) ASTER: The Advanced Spaceborne Thermal Emission and Reflection Radiometer (ASTER) is a 1 arc-second global DEM [22]. The ASTER version 3 has been publically released in 2019 (download from e.g. https://asterweb.jpl.nasa.gov/gdem.asp). Table 2 shows the characteristics of those selected global DEMs.

Table 2 Characteristics of the utilized global DEMs

\begin{tabular}{|c|c|c|c|}
\hline \multirow{2}{*}{ DEM } & \multirow{2}{*}{ Released year } & \multicolumn{2}{|c|}{ Spatial resolution } \\
\cline { 3 - 4 } & & arc second & $\mathrm{m}$ \\
\hline ASTER v.3 & 2019 & $1 "$ & $\sim 30$ \\
\hline ACE2 & 2019 & $3 "$ & $\sim 90$ \\
\hline SRTMGL1 v.3 & 2019 & $1 "$ & $\sim 30$ \\
\hline
\end{tabular}

The topography of Egypt, as derived from ACE2 GDEM for example, is depicted in Fig. 1. It can be noticed that the Egyptian terrain heights range from $-137 \mathrm{~m}$ to $+2603 \mathrm{~m}$ with an average of $302 \mathrm{~m}$. It can be realized that the mountainous areas are located only in the middle of Sinai peninsula, eastern deserts along the Red sea, and in Southwestern borders. The flat topography generally reduces the effects of terrain corrections in geoid modelling. For each combination, a 5'x5' gravimetric geoid is developed first, and then fitted to GNSS/levelling dataset.

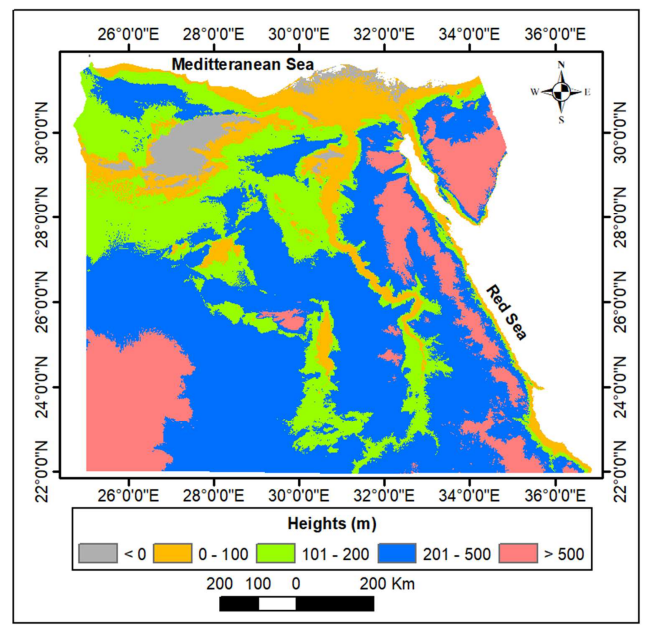

Fig. 1 Topography of Egypt 
The least-square collocation is one of the most imperative tools for gravity field modelling due to its high capability of dealing with heterogeneous input features. The basic mathematical form of LSC, in a vector form, could be written as [23]:

$$
x=A u+s+n
$$

where $x$ is the vector of measurements, $n$ is the vector of the noise component in the measurements, $s$ is the vector of signal components in the measurements to be predicted, and $u$ is the vector of unknown parameters to be estimated.

Thus, LSC combines adjustment, filtering and prediction of the input Earth's gravity field parameters to estimate geoidal heights and other geodetic related values [ibid]. Solving that model requires constructing a covariance matrix that can be estimated based on the variances of the required noise and unknown parameters. The accuracy of LSC solution, as represented by the error covariance matrix, depends on the original covariance matrices or, in other words, on the accuracy of the input datasets.

The processing, in the current study, has been carried out using the LSC-based Gravsoft package [24]. With this software, the effects of a GGM and a DEM are first removed from the input datasets of the Earth's gravitational field to obtain a residual field, second computations of predicted quantities and error estimates are carried out, and finally re-adding the effects of both GGM and DEM to the attained residual parameters [23]. Additionally, an empirical covariance function is estimated based on the residual gravity anomaly data. The package decomposes the geoid undulations $(\mathrm{N})$, in the remove-compute-restore processing strategy, into three components as [24]:

$$
N=N_{G G M}+N_{\Delta g}+N_{T C}
$$

where $N_{G G M}$ provides the long to the medium wavelength of the gravitational field of the Earth as computed, $N_{\Delta g}$ represents the medium to short local wavelength deduced from local datasets, and $N_{T C}$ represents the terrain corrections or the topography contribution as represented by a certain DEM. The three components of the geoidal undulation, in the last equation, are computed by the following equations [23]:

$$
\begin{aligned}
& N_{G G M}=\left(\frac{G M}{r \gamma}\right) \sum_{n=2}^{\max -n}\left(\frac{a}{r}\right)^{n} \sum_{m=0}^{n}\left(\left(\bar{C}_{n m} \cos m \lambda+\bar{S}_{n m} \sin m \lambda\right) P_{n m} \cos \varphi\right) \\
& N_{\Delta g}=\frac{R}{4 \pi \gamma} \int_{\sigma} \int_{o} S(\psi)\left(\Delta g-\Delta g_{G G M}\right) d \sigma \\
& N_{T C}=G \iint_{\sigma} \int_{h_{p}}^{h} \frac{\rho(x, y, z)\left(h_{p}-z\right)}{r^{3}\left(x_{p}-x, y_{p}-y, h_{p}-z\right)} d x d y d z
\end{aligned}
$$

where $n$ is the degree of the GGM model, max- $n$ is the maximum degree of the GGM model, $m$ is the maximum order of the model, $\gamma$ is the normal gravity of the reference ellipsoid, $r$ is the geocentric radius of the computation point, $G$ is the Newtonian gravitational constant, $M$ is the mass of the Earth, $R$ is the mean radius of the Earth, $a$ is the semi-major axis, $\varphi$ is the geocentric latitude, $\lambda$ is the geocentric longitude, $\Delta g$ is the gravimetric observations given as free-air gravity anomalies, $\Delta g_{G G M}$ is the gravity anomalies computed by a specific GGM, $S(\Psi)$ is the Stokes' function, $\sigma$ is a differential surface area on a unit sphere, $\sigma \circ$ is the differential surface area on the computational cap, $\bar{C}_{n m}$ and $\bar{S}_{n m}$ are the fully normalized harmonic coefficients, $\rho_{\circ}$ is the surface atmosphere potential, $r_{0}$ is the sea-level radius of the Earth, $\rho(x, y, z)$ is the topographical density at running point, $h_{p}$ and $h$ are the computational and running point respectively, and $P_{n m}$ is the fully normalized associated Legendre polynomial. 
Next, all geoid combinations have been investigated using the ArcGIS 10 software, to interpolate the attained geoidal heights at the 100 checkpoints and compare them to their corresponding known values. To compare the performance of each attained LGM, three statistical measures have been used: standard deviation, average, and range of the discrepancies between the known and interpolated geoid undulations. Three descending ranks $(R 1, R 2, R 3)$ are given to each LGM over a scale of ten, where ten represents the minimum value of the statistical measure and one represents the maximum value. Weights of those ranks have been selected as 4,3 , and 3 respectively. Hence, a weighted mean rank $(R)$ is computed for each LGM, using its corresponding three individual rank measures $(R i)$ and their assigned weights $(W i)$ to represent a unique indicator of its accuracy and to serve as a base of comparison between the twelve accomplished LGMs, as:

$$
R=\frac{\sum R_{i} W_{i}}{\sum W_{i}}
$$

Fig. 2 presents the overall steps utilized in the data processing of the proposed methodology.

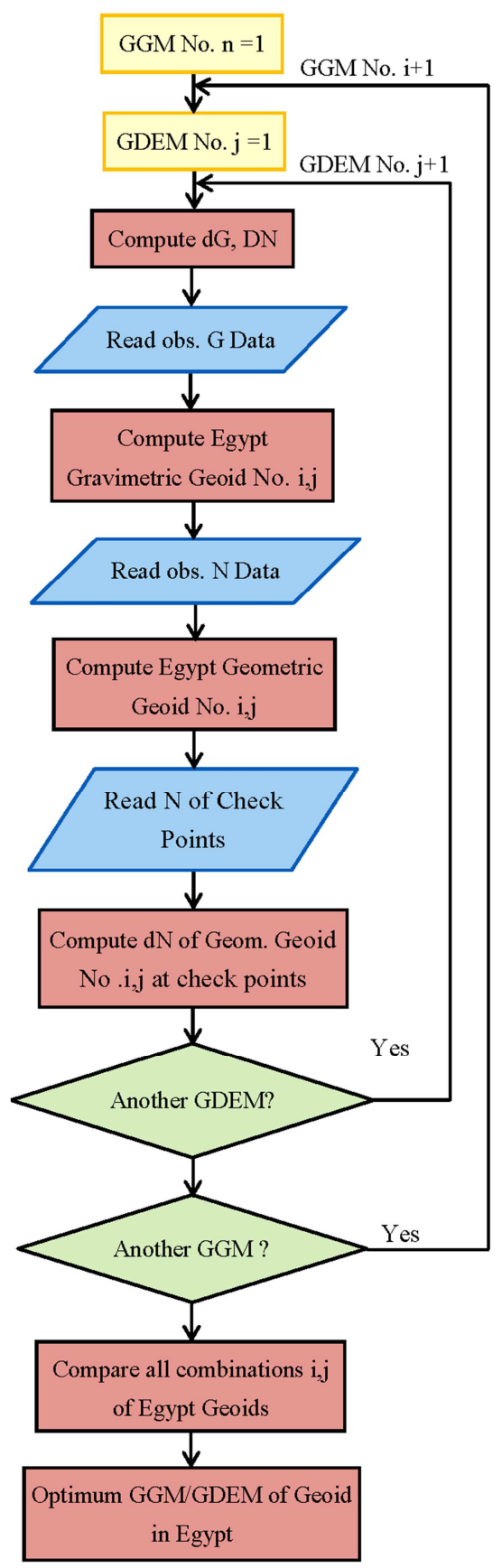

Fig. 2 Flow chart of data processing 


\section{Available Data}

Regarding the available terrestrial data over Egypt, the study utilized two main types: terrestrial gravity data and GNSS/Levelling data (Fig. 3). The first-order Egyptian national gravity networks of both 1997 and 1977 contain 247 measured gravity points [11]; and 1100 GNSS/levelling stations all over Egypt, that were observed by the Survey Research Institute (SRI) in various projects over the last ten years [25]. It is worth mentioning that the average accuracy of the Egyptian National Gravity Standardization Network of 1997 (ENGSN97) is $\pm 0.02 \mathrm{mGal}$, while the corresponding value of the National Gravity Standard Base Network of 1977 (NGSBN77) is $\pm 0.08 \mathrm{mGal}$ [11]. On the other hand, the utilized GNSS/levelling points have been surveyed using the first-order levelling and the first-order GNSS geodetic network standards, and their average accuracy could be estimated as \pm 3 - $4 \mathrm{~cm}$ [25]. Ten percent of the known GNSS/Levelling stations have been reserved as checkpoints to estimate the external accuracy of each developed LGM. It should be noticed that such local datasets may not adequate in number and they are not homogeneously distributed over Egypt. However, such data are the most-recent accurate geodetic measurements existing over the country [ibid].

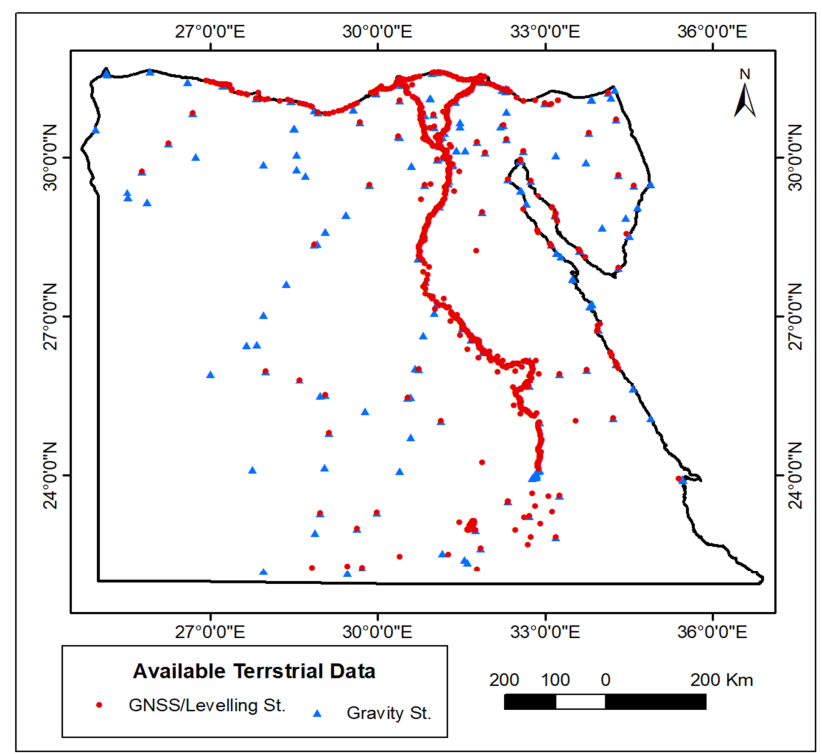

Fig. 3 Available terrestrial data in Egypt

\section{Results and Discussion}

Table 3 Overall accuracy of different GGMs and DEMS over checkpoints (m)

\begin{tabular}{|c|c|c|}
\hline (A) Overall accuracy of LGMs based on different GGMs over checkpoints: \\
\hline GGM & Average Standard Deviation (m) & Improvements \% \\
\hline XGM2019e_2159 & \pm 0.137 & \multirow{2}{*}{$17 \%$} \\
\hline EGM2008 & \pm 0.140 & \\
\hline GECO & \pm 0.159 & \multirow{2}{*}{$2 \%$} \\
\hline EIGEN-6C4 & \pm 0.157 & \multirow{2}{*}{$2 \%$} \\
\hline (B) Overall accuracy of LGMs based on different DEMs over checkpoints: \\
\hline DEM & Average Standard Deviation (m) & Improvements \% \\
\hline ACE2 & \pm 0.146 & \\
\hline ASTER v.3 & \pm 0.148 & \\
\hline SRTMGL1 v.3 & \pm 0.150 & \\
\hline
\end{tabular}

The first investigation step has been carried out in the overall perspective to get the big picture about the contribution of GGMs and DEMs in geoid modelling for Egypt. Hence, the average standard deviation over checkpoints for each GGM-based LGM, averaging its performance using all DEMs, has been computed. Similarly, the mean standard deviation over check points for each DEM-based LGM, averaging its performance using all GGMs, has been also computed. Table 3 presents the accomplished findings that reveal two imperative remarks. First, local geoid accuracy levels range from $\pm 0.137 \mathrm{~m}$ using the 
XGM2019e_2159 model to $\pm 0.159 \mathrm{~m}$ using the GECO GGM. It emphasizes that significant improvements of $17 \%$ approximately in local geoid accuracy are attributed to the utilization of GGMs models representing the long wavelength of the Earth gravitational field. Regarding the contribution of DEMs, it can be noticed from that table that the overall accuracy of LGMs does not significantly depend on the utilized DEM. That could be attributed to the almost flat topography of Egypt as depicted in Fig. 1, which reduces the effect of the topographic correction in the geoid modelling process.

Furthermore, the statistical characteristics of the developed twelve LGM of Egypt are tabulated in Tables 4-7. Several imperative remarks could be perceived from those tables. First, it can be recognized, from the first column in all tables, that the

Table 4 Chrematistics of XGM2019e_2159 GGM differences over checkpoints (m)

\begin{tabular}{|c|c|c|c|}
\hline \multirow{2}{*}{ Measure/LGM No. } & \multicolumn{3}{|c|}{ Utilized GDEM } \\
\cline { 2 - 4 } & ACE2 & ASTER v.3 & SRTMGL1 v.3 \\
\cline { 2 - 4 } & 1 & 2 & 3 \\
\hline Minimum & -0.276 & -0.279 & -0.275 \\
\hline Maximum & 0.271 & 0.430 & 0.429 \\
\hline Range & 0.547 & 0.710 & 0.704 \\
\hline Average & 0.003 & 0.005 & 0.006 \\
\hline Standard Deviation & \pm 0.129 & \pm 0.140 & \pm 0.141 \\
\hline
\end{tabular}

Table 5 Chrematistics of GECO GGM differences over checkpoints (m)

\begin{tabular}{|c|c|c|c|}
\hline \multirow{2}{*}{ Measure/LGM No. } & \multicolumn{3}{|c|}{ Utilized GDEM } \\
\cline { 2 - 4 } & ACE2 & ASTER v.3 & SRTMGL1 v.3 \\
\cline { 2 - 4 } & 4 & 5 & 6 \\
\hline Minimum & -0.292 & -0.301 & -0.293 \\
\hline Maximum & 0.649 & 0.607 & 0.663 \\
\hline Range & 0.940 & 0.908 & 0.956 \\
\hline Average & 0.016 & 0.015 & 0.017 \\
\hline Standard Deviation & \pm 0.161 & \pm 0.156 & \pm 0.162 \\
\hline
\end{tabular}

Table 6 Chrematistics of EIGEN-6C4 GGM differences over checkpoints (m)

\begin{tabular}{|c|c|c|c|}
\hline \multirow{2}{*}{ Measure/LGM No. } & \multicolumn{3}{|c|}{ Utilized GDEM } \\
\cline { 2 - 4 } & ACE2 & ASTER v.3 & SRTMGL1 v.3 \\
\cline { 2 - 4 } & 7 & 8 & 9 \\
\hline Minimum & -0.621 & -0.633 & -0.633 \\
\hline Maximum & 0.369 & 0.375 & 0.376 \\
\hline Range & 0.989 & 1.008 & 1.008 \\
\hline Average & 0.010 & 0.010 & 0.010 \\
\hline Standard Deviation & \pm 0.156 & \pm 0.157 & \pm 0.157 \\
\hline
\end{tabular}

Table 7 Chrematistics of EGM2008 GGM differences over checkpoints (m)

\begin{tabular}{|c|c|c|c|}
\hline \multirow{2}{*}{ Measure/LGM No. } & \multicolumn{3}{|c|}{ Utilized GDEM } \\
\cline { 2 - 4 } & ACE2 & ASTER v.3 & SRTMGL1 v.3 \\
\cline { 2 - 4 } & 10 & 11 & 12 \\
\hline Minimum & -0.295 & -0.317 & -0.320 \\
\hline Maximum & 0.322 & 0.327 & 0.328 \\
\hline Range & 0.618 & 0.644 & 0.647 \\
\hline Average & 0.019 & 0.018 & 0.019 \\
\hline Standard Deviation & \pm 0.140 & \pm 0.140 & \pm 0.140 \\
\hline
\end{tabular}


AEC2 GDEM generally produce the minimum range of variations over checkpoints. So, it might be concluded that AEC2 performs a little bit better than the other two GDEMs over Egypt, at least in geoid modelling applications. Moreover, it can be revealed that the standard deviation, as an accuracy indicator, ranges from $\pm 0.129 \mathrm{~m}$ for LGM 1 to $\pm 0.162 \mathrm{~m}$ for LGM 6 . For the range of discrepancies over known checkpoints, the developed LGMs perform diversely and their variations range from a minimum of $0.547 \mathrm{~m}$ for LGM 1 to $1.008 \mathrm{~m}$ for LGM 8 and 9. However, all these levels of accuracy are not accurate enough for high-precision GNSS-based geodetic and mapping applications in Egypt. Consequently, this signifies the critical need for collecting more measured geodetic datasets in terms of number and homogenous distribution over the country.

To come up with the optimum LGM for Egypt, the twelve LGMs have been compared in terms of standard deviation, average, and ranges of their geoid undulations' variations over checkpoints. As mentioned earlier, a descending rank is assigned to each LGM, on a scale of ten, for each statistical measure. Subsequently, Eq. 2 is applied to compute the weighted mean rank for each model. The attained results are tabulated in Table 8. It can be realized that the overall rank of the LGMs, on a scale of ten, varies from 2.9 for LGM 6 to 10.0 for LGM 1. Consequently, based on the currently available local geodetic datasets, LGM 1 is the optimum model for Egypt. It has been developed based on the XGM2019e_2159 GGM and the ACE2 GDEM. Its geoid undulations (Fig. 4) range from $8.948 \mathrm{~m}$ to $21.088 \mathrm{~m}$ with a mean of $14.106 \mathrm{~m}$. It is recommended to use this local geoid, for the time being, for GNSS-based surveying and mapping applications in Egypt.

Table 8 Ranks of developed LGMs for Egypt

\begin{tabular}{|c|c|c|c|c|}
\hline LGM No. & $\mathrm{R}_{1}$ & $\mathrm{R}_{2}$ & $\mathrm{R}_{3}$ & $\mathrm{R}$ \\
\hline 1 & 10 & 10 & 10 & 10.0 \\
\hline 2 & 9 & 9 & 1 & 7.2 \\
\hline 3 & 8 & 8 & 2 & 6.8 \\
\hline 4 & 3 & 5 & 5 & 4.0 \\
\hline 5 & 7 & 6 & 4 & 6.1 \\
\hline 6 & 1 & 4 & 6 & 2.9 \\
\hline 7 & 6 & 7 & 3 & 5.7 \\
\hline 8 & 4 & 7 & 3 & 4.7 \\
\hline 9 & 4 & 7 & 3 & 4.7 \\
\hline 10 & 9 & 2 & 8 & 6.7 \\
\hline 11 & 9 & 3 & 7 & 6.8 \\
\hline 12 & 9 & 2 & 9 & 6.9 \\
\hline
\end{tabular}

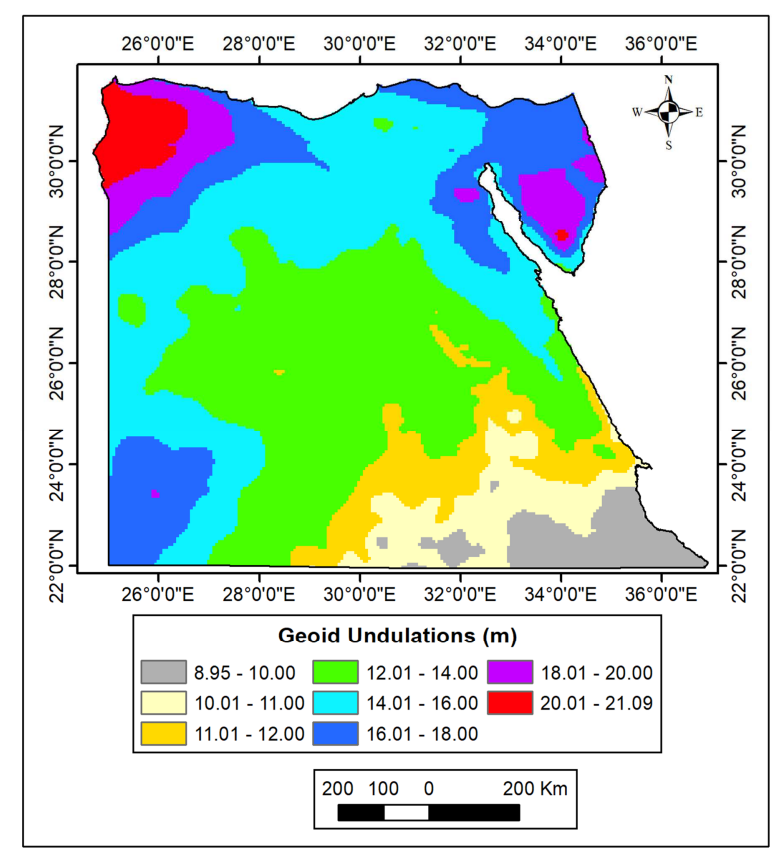

Fig. 4 Developed optimum local geoid of Egypt 


\section{Conclusions}

Local geoid modelling involves a local geodetic dataset, an optimum GGM, and a GDEM. A precise geodetic dataset, comprising 247 terrestrial gravity points and 1000 GNSS/Levelling stations, has been utilized in the current research study using the Least-Square Collocation geoid modelling method. Rather than the classical point-wise method of investigating the accuracy of GGMs and DEMs, the current research proposed a new approach for deciding which GGM and DEM models are optimum for utilization in developing a national geoid model. Thus, four GGMs (namely XGM2019e_2159, EGM2008, EIGEN-6C4, and GECO) and three GDEMs (ASTER v.3, ACE2, and SRTMGL1 v.3) have been utilized and twelve 5'x5' gravimetric geoid models have been developed for Egypt. Such models have been, then, fitted to GNSS/Levelling stations and then judged over 100 checkpoints with known geoidal heights.

Accomplished findings revealed that the utilized GDEMs provide comparable results from a precision perspective, which could be due to the flat topography of Egypt. Thus, it can be concluded that the terrain effects in geoid modelling are quite small. Additionally, it has been found that the AEC2 model performs relatively better than the other two investigated GDEMs over Egypt. Furthermore, it has been found that the standard deviation of the twelve LGM ranges from $\pm 0.129 \mathrm{~m}$ to $\pm 0.162 \mathrm{~m}$. On the other hand, the undulations discrepancies over known checkpoints vary from $0.547 \mathrm{~m}$ to $1.008 \mathrm{~m}$. Concerning the general performance of all investigated GGMs, it has been concluded that the XGM2019e_2159 produces the best standard deviation ( $\pm 0.129 \mathrm{~m}$ ) while the GECO GGM produces the worst value $\pm 0.162 \mathrm{~m}$ ). Nevertheless, all these levels of accuracy cannot be as accurate as needed for high-precision GNSS-based geodetic and mapping applications in Egypt. As a result, there is a vital need for collecting extra local geodetic datasets in terms of number and homogenous distribution over the country. All specialized governmental, military and private organizations have to cooperate together to establish a recent integrated national dataset for geodetic and environmental applications.

Based on statistical ranking, it has been found that the LGM developed using the XGM2019e_2159 GGM and the ACE2 GDEM, could be considered as the optimum model for Egypt for the time being. Therefore, it is recommended to use this local geoid for GNSS-based surveying and mapping applications in Egypt.

\section{Acknowledgement}

The authors appreciated the revision of the manuscript and the valuable comments given by the late Prof. Dalal S. Alnaggar who passed away during the development of this research study. May God blesses her soul.

\section{Conflicts of Interest}

The authors declare no conflict of interest.

\section{References}

[1] K. M. Ahlgren, S. A. Holmes, X. Li, Y. M. WANG, and M. A. Youngman, "Geoid Modeling at NOAA' s National Geodetic Survey as 2022 Approaches," FIG Working Week, Helsinki, Finland, 2017.

[2] M. Greaves, P. Downie, and K. Fitzpatrick, "OSGM15 and OSTN15: Updated Transformations for UK and Ireland," Geomatics World, No. 32/33, pp. 1-5, 2016.

[3] F. Duquenne and A. Coulomb, "The French Approach to Modernize Its Vertical Reference," Finance Information Group Working Week, May 2015, pp. 17-21.

[4] K. Matsuo and Y. Kuroishi, "Refinement of a Gravimetric Geoid Model for Japan using GOCE and An Updated Regional Gravity Ffield Model," Earth, Planets and Space, vol. 72, no. 1, pp. 1-18, March 2020.

[5] A. V. Fernádez, O. L. Castro, and J. G. León, "Geoid Heights in Costa Rica, Case of Study: Central Pacific Zone," Revista Ingenieria, vol. 30, no. 1, pp. 1-20, November 2020. 
[6] M. El-Ashquer, H. Al-Ajami, A. Zaki, and M. Rabah, "Study on the Selection of Optimal Global Geopotential Models for Geoid Determination in Kuwait," Survey Review, vol. 52, no. 373, pp. 373-382, May 2019.

[7] M. E. Gomez, R. Perdomo, and D. Del Cogliano, "Validation of Recent Geopotential Models in Tierra Del Fuego," Acta Geophysica, vol. 65, no. 5, pp. 931-943, September 2017.

[8] A. A. Saad and M. S. Elsayed, "Evaluation of GOCE Satellite Only Models Along the River Nile," Scientific and Academic Publishing, vol. 8, no. 3, pp. 131-140, 2019.

[9] M. Eshagh and S. Zoghi, "Local Error Calibration of EGM08 Geoid using GNSS/levelling Data,” Journal of Applied Geophysics, vol. 130, pp. 209-217, July 2016.

[10] D. S. Alnaggar, “Determination of the Geoid in Egypt Using Heterogeneous Geodetic Data," PhD dissertation, Faculty of Engineering, Cairo University, 1986.

[11] G. M. Dawod, “A National Gravity Standardization Network for Egypt," PhD dissertation, Faculty of Engineering at Shoubra, Zagazig University, 1998.

[12] H. Abd-Elmotaal, "Gravimetric Geoid for Egypt Using High-Degree Tailored Reference Geopotential Model," National Research Institute of Astronomy and Geophysics Journal of Geophysics. Special issue, pp. 507-531, December 2008.

[13] G. Dawod, W. Resrach, and T. M. Abdelaziz, "Utilization of Geographically Weighted Regression for Geoid Modelling in Egypt,” Journal of Applied Geodesy, vol. 14, no. 1, October, 2019.

[14] T. Abdel-Aziz, G. Dawod, and H. Ebaid, "DEMs and Reliable Sea Level Rise Risk Monitoring in Nile Delta, Egypt," Discover Sustainability, vol. 1, no. 1, pp. 1-11, December 2020.

[15] P. Zingerle, R. Pail, and T. Gruber, "High-Resolution Combined Global Gravity Field Modelling--Towards a Combined d/o 10800 Model," Geophysical Research Abstracts, vol. 21, January 2019.

[16] M. Gilardoni, M. Reguzzoni, and D. Sampietro, "GECO: A Global Gravity Model by Locally Combining GOCE Data and EGM2008," Studia Geophysica et Geodaetica, vol. 60, no. 2, pp. 228-247, March 2016.

[17] C. Förste, S. Bruinsma, O. Abrikosov, F. Flechtner, J. C. Marty, J. M. Lemoine, et al., "EIGEN-6C4-The Latest Combined Global Gravity Field Model Including GOCE Data up to Degree and Order 1949 of GFZ Potsdam and GRGS Toulouse," EGUGA, p. 3707, May 2014.

[18] N. K. Pavlis, S. A. Holmes, S. C. Kenyon, and J. K. Factor, "The Development and Evaluation of the Earth Gravitational Model 2008 (EGM2008)," Journal of Geophysics Research Solid Earth, vol. 117, no. B4, April 2012.

[19] “The International Center for Global Earth Model," http://icgem.gfz-potsdam.de/tom_longtime, October 12, 2020.

[20] “SRTMGL1 V003,” The US Geological Survey, https://lpdaac.usgs.gov/products/srtmgl1v003/, 2020.

[21] R. Smith and P. Berry, "Altimeter Corrected Elevations, Version 2," https://sedac.ciesin.columbia.edu/data/set/dedc-ace-v2, 2019.

[22] “ASTER Digital Elevation Model V003," https://catalog.data.gov/dataset/aster-digital-elevation-model-v003, July 17, 2020.

[23] B. Hofmann-Wellenhof, "Moritz, H.(2005) Physical Geodesy”, New York: Springer, 1967.

[24] R. Forsberg and C. C. Tscherning, GRAVSOFT: Geodetic Gravity Field Modelling Programs Manual, 2nd ed. Denmark: National Space Institute, 2008.

[25] E. M. Al. Krargy, “Development of a National Geoid for Egypt Using Recent Surveying Data,” PhD dissertation, Faculty of Engineering, Minufiya university, Egypt, 2016.

Copyright $(\mathrm{C}$ by the authors. Licensee TAETI, Taiwan. This article is an open access article distributed under the terms and conditions of the Creative Commons Attribution (CC BY-NC) license (https://creativecommons.org/licenses/by-nc/4.0/). 